\title{
Los antibióticos proveen escaso beneficio en los niños con otitis media aguda
}

\section{Objetivo}

Determinar la efectividad del uso de antibióticos en niños con otitis media aguda (OMA), en términos de alivio de los síntomas, presencia de complicaciones y trastornos auditivos a mediano y largo plazo.

\section{Diseño}

Revisión sistemática de los estudios aleatorizados de alta calidad metodológica que comparan el uso de antibióticos versus placebo en niños con OMA. Los autores revisaron la biblioteca Cochrane, Medline, Current Contents, y la bibliografía de los artículos, desde el año 1958 a 1999 inclusive.

\section{Selección de estudios}

De un total de 10 estudios, se seleccionaron 7 que contenían resultados relevantes, con un total de 2202 niños.

\section{Resultados principales}

Dolor: A las 24 horas de iniciado el tratamiento, dos tercios de los niños ya no tuvieron dolor, independientemente de estar recibiendo placebo o antibiótico. Un $80 \%$ de los pacientes del grupo control presentaron reducción espontánea del dolor entre el $2^{\circ}$ y $7^{\circ}$ día. El grupo que recibió antibiótico tuvo una reducción relativa de riesgo de dolor del $28 \%$ (IC95\% 15-38\%) y una reducción absoluta de riesgo del $5 \%$. Esto significa que se necesita tratar a 17 niños con antibiótico para prevenir que uno de ellos tenga dolor después del segundo día de tratamiento (NNT=17).

Sordera: No hubo diferencias clínicas ni estadísticas significativas en los resultados de la timpanometría luego de 1 a 3 meses del episodio agudo.

Progresión de los síntomas y efectos adversos: En el grupo tratado se encontró una tendencia no estadísticamente significativa hacia el menor desarrollo de otitis contralateral, y un mayor número de efectos adversos (náuseas, diarrea y rash). No hubo diferencia significativa en el número de recurrencias entre ambos grupos.

Complicaciones: Ocurrieron pocas complicaciones serias, en ambos grupos. Hubo sólo un caso de mastoiditis, en un grupo tratado con penicilina., por lo que la aplicabilidad de estos hallazgos en grupos en donde la mastoiditis es frecuente, es incierta.

\section{Conclusiones}

El uso de antibióticos acorta el curso de la OMA en niños. Sin embargo, la mayoría de los casos resolverán espontáneamente sin complicaciones asociadas. Este beneficio debería cotejarse con la posibilidad de reacciones adversas asociadas a los mismos. El uso de antibióticos podría tener una función importante en la reducción del riesgo de mastoiditis, en aquellas poblaciones donde esta entidad es más frecuente. Caso contrario, en el manejo de estos pacientes debería enfatizarse el uso de una adecuada analgesia, limitando el uso de antibióticos. Se necesitan nuevos estudios para identificar a subgrupos con evolución prolongada o complicada, así como la realización de revisiones sistemáticas acerca del rol de diferentes tratamientos y medidas preventivas (como vacunas o xylitol).

Fuente de financiamiento: Sin conflictos de interés.

\section{Comentario}

La OMA es la enfermedad más comúnmente diagnosticada en pediatría y causa número uno de prescripción antibiótica. Su óptimo tratamiento permanece abierto a constante controversia. ${ }^{1.3}$

Los episodios de otitis han sido tradicionalmente tratados con antibióticos, principalmente por tres motivos: 1) Las causas son mayoritariamente bacterianas, con el neumococo como principal exponente; 2) Más rápida mejoría sintomática y de la infección, si bien existe una tasa alta de resolución espontánea; y 3) Prevención de las complicaciones supurativas.

Este metaanalisis muestra que una minoría de los niños se beneficiará con los antibióticos. Debido que al inicio no es posible saber cual los necesitará y cual no, se plantea la opción de tratar los síntomas sin antibióticos, y recién agregarlos de no observar mejoría en el seguimiento.

En Holanda, se aplica esta estrategia desde 1990. Varios países de Europa se han sumado y recientemente, la Academia Americana de Pediatría ha realizado una guía de práctica clínica incorporándola. ${ }^{4}$

Esta opción se refiere a la posibilidad de diferir el tratamiento antibiótico por 48-72 horas en determinados niños, limitando el manejo al tratamiento sintomático. La decisión de observar o tratar está basada en la edad del niño, el diagnóstico de certeza, la severidad de la enfermedad y la factibilidad de seguimiento del paciente.

Las diferentes sociedades y academias de pediatría difieren en estos límites. En general, se acepta que en los casos de niños ma- yores de 6 meses y menores de 2 años con otitis leve a moderada, sin historia de OMA recurrente, podría diferirse inicialmente el uso de antibióticos, y utilizarlos si no hay mejoría clínica a las 48-72 horas de tratamiento. Esta conducta podría hacer disminuir el uso indiscriminado de antibióticos, y consecuentemente la preocupante elevación de las tasas de resistencia antibiótica bacteriana y los efectos adversos de los mismos. La mayoría de las guías de práctica indican el tratamiento antibiótico de inicio en los menores de 6 meses.

Algunos autores han expresado su preocupación acerca de un posible resurgimiento de la mastoiditis con esta estrategia. Sin embargo, el reporte de la Agencia para la investigación y calidad en el cuidado de la salud de EE.UU. (AHRQ) ${ }^{5}$ concluyó que la mastoiditis no aumenta con la estrategia de observación inicial, siempre y cuando los niños sean seguidos de cerca, y el tratamiento antibiótico sea iniciado en aquellos que no mejoren.

\section{Conclusiones del comentador:}

La estrategia de tratar los síntomas relacionados a OMA sin antibioticoterapia, y agregar antibióticos si no se observa mejoría a las 48hs. puede ser utilizada en los mayores de 6 meses con OMA leve a moderada y sin antecedentes de OMA recurrente. Además, debe ser una población en la que se pueda asegurar un seguimiento cercano por un adulto responsable, y acceso a la atención médica en cualquier momento del seguimiento.

\section{Dra. Erica Rodríguez Gesto [ Instructora Hospital de Niños R. Gutiérrez. ]}

Rodríguez Gesto E. Los antibióticos proveen escaso beneficio en los niños con otitis media aguda. Evid. actual. práct. ambul. 2004;7:166. Comentado de: Antibiotics for acute otitis media in children. Glasziou PP, Del Mar CB, Sanders, SL, Hayem M. Cochrane Review. In: The Cochrane Library, Issue 3, 2003.

\section{Referencias}

1.Paradise, J Otitis Media. Nelson Textbook of Pediatrics 17th Edition. 2003. Pág: 2138-2149.

2.Damoiseaux R, Van Balen F, et al. Primary care based randomized, double blind trial of amoxicilin versus placebo for acute otitis media in children aged under 2 year. BMJ 2000; 320:350-4

3.Del mar C, Glaziou P et al. Are antibiotics indicated as initial treatment for children whith acute otitis media? A meta-analysis. BMJ 1997;314: 15216-9.

4.AAP. Subcommittee on Management of Acute Otitis Media, American Academy of Pediatrics. Diagnosis and Management of Acute Otitis Media. Pediatrics. Mayo 2004; Vol 113: 14511465.

5.Marcy M, Takata G et al. Management of acute otitis media. Evidence report/technology assessment ํㅜ15. AHRQ publication № 01-E010 Rockville, MD: agency for healthcare Reserch and Quality; 2001 